\title{
A brief historical overview of artificial intelligence research
}

\author{
Stacey Tobin ${ }^{\mathrm{a}}$, Bamini Jayabalasingham ${ }^{\mathrm{b}, *}$, Sarah Huggett ${ }^{\mathrm{c}}$ and Maria de Kleijn ${ }^{\mathrm{d}}$ \\ ${ }^{a}$ Biomedical Writer and Editor, Elsevier, 230 Park Avenue Suite 800, New York, NY 10169, USA \\ ${ }^{\mathrm{b}}$ Senior Analytical Product Manager, Elsevier, 230 Park Avenue Suite 800, New York, NY 10169, USA \\ ${ }^{\mathrm{c}}$ Analytical Services Manager, Elsevier, 230 Park Avenue Suite 800, New York, NY 10169, USA \\ ${ }^{\mathrm{d}}$ Senior Vice President, Analytical Services, Elsevier, 230 Park Avenue Suite 800, New York, NY 10169, \\ USA
}

\begin{abstract}
This paper gives a brief timeline of the evolution of the field of Artificial Intelligence (AI), a field that is currently generating approximately sixty thousand research papers per year. This represents a $12.9 \%$ growth in output over the past five years, and is markedly higher than the growth seen across all research, which has grown at a rate of $2.3 \%$ per year in the same period. AI research is of interest around the world and this paper highlights many of the activities undertaken to stimulate this research in the USA, Canada, Mexico, Europe, and Asia.
\end{abstract}

Keywords: Artificial intelligence, AI policies, history of AI, global AI strategies

\section{Introduction}

Historical timelines of research in the field of Artificial Intelligence (AI) describe a sixty-year journey, including "AI winters" [1] (understood as periods of disillusionment with the technology) and breakthroughs. In Fig. 1, we plot a few of the significant events, policies, and breakthroughs in the field of AI. In terms of technological advances, research in neural networks started winning vision and speech competitions in 2005, and was a dominant subfield of research by 2009 [2].

In terms of scholarly communication, several good review (survey) papers on deep learning started to appear around 2014-2015 [3]. The development of the AI field in the last two decades has been described by both the Think Tank Eurasia Group, Sinovation Ventures [4], and Dr. Kai-Fu Lee [5] as occurring in four phases of five-years each, focusing on: Internet AI (recommender systems), Business AI (fraud detection, financial forecasting), Perception AI (smart devices), and Autonomous AI (new hardware applications, such as self-driving cars).

Counting scholarly publications is a common and easily understood measurement of research output. What follows is an overall view on all types of scholarly publications in the field of AI that are indexed in Scopus [6], namely journal articles, conference papers, and others, such as literature reviews or short communications. The following analysis is based on a refined corpus of more than six hundred thousand

\footnotetext{
${ }^{*}$ Corresponding author. E-mail: b.jayabalasingham@elsevier.com.
} 


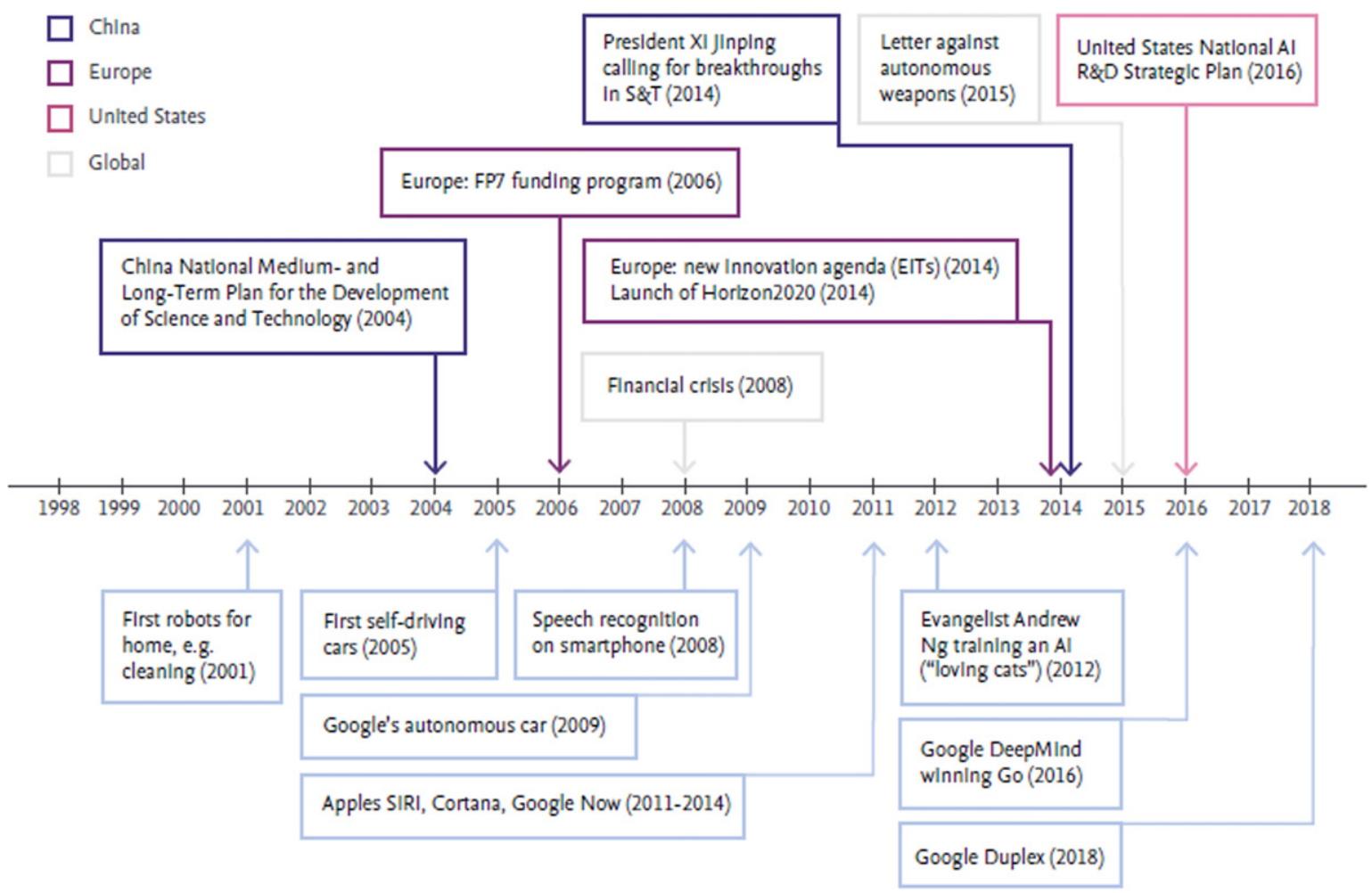

Fig 1. Timeline of policies, events, and breakthroughs of significance in the AI field.

AI publications from 1998 to 2017, retrieved from Scopus in May 2018 [7]. The graph in Fig. 2 illustrates the overall growth of the AI research field, which now generates approximately sixty thousand publications per year. Globally, the field of AI has shown strong growth of $12.9 \%$ per year in the last five years. This growth is markedly higher than the growth seen across all research, which has grown at a rate of $2.3 \%$ per year in the same period. The decreased cost of computing power paired with increased availability of large data sets for training machines is responsible for this high growth rate.

\subsection{Overview of recent national and regional AI strategies}

Developing capacity for AI research, technology, and application is increasingly perceived as essential to national competitiveness, security, and economic strength. In the last few years, several countries and regions have developed and released AI strategic plans as they race towards global leadership [8]. These strategies usually contain at least one of three frequent components:

- Recommendations to invest in building the AI workforce and research and development.

- Suggestions on how to respond to the ways in which AI is likely change jobs and economies.

- Analysis of the social, economic, and ethical implications of AI. 


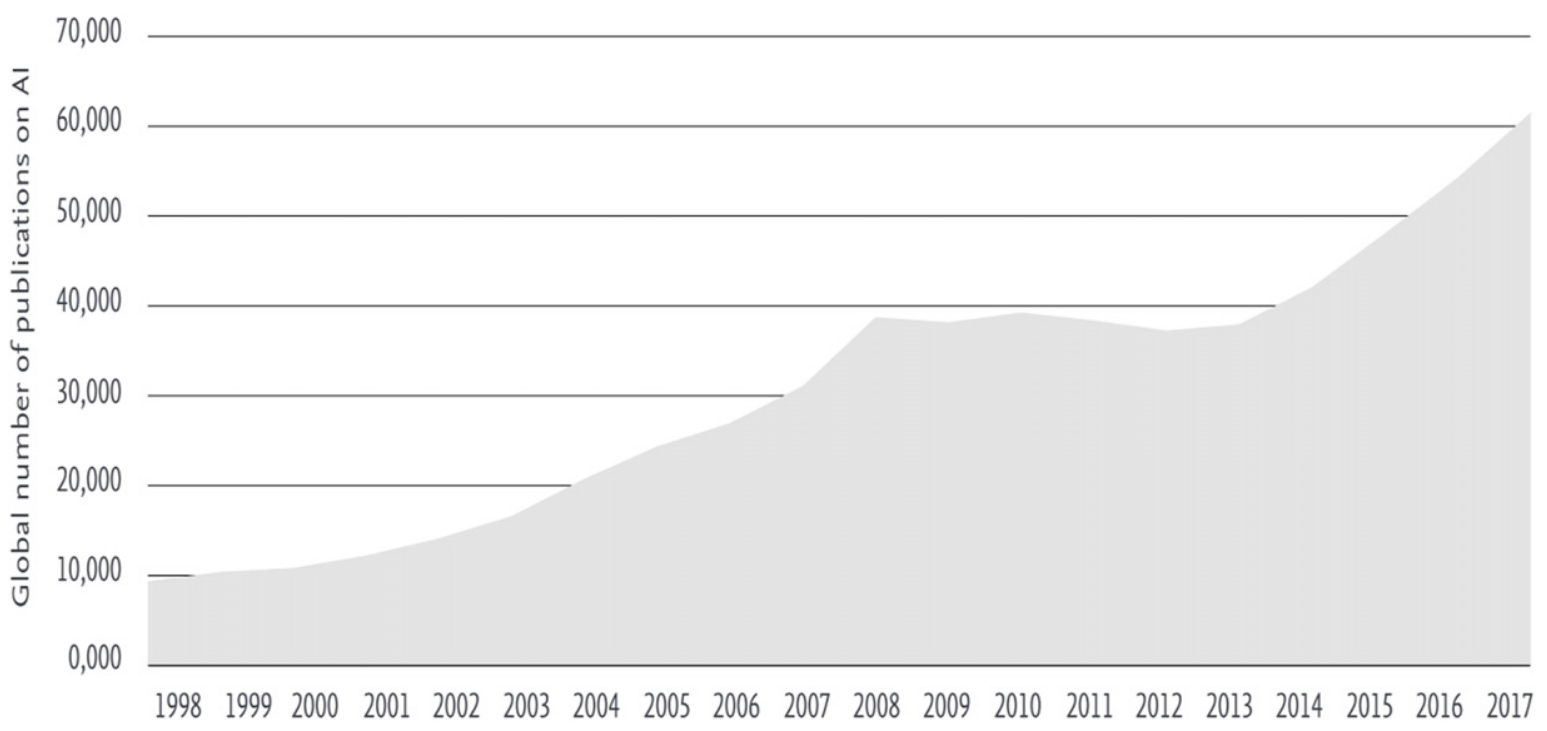

Fig 2. Annual number of AI publications, all document types, 1998-2017. Source: Scopus.

\subsection{Americas: The United States (USA) shows early and continued interest in AI}

In October 2016, the National Science and Technology Council released a report entitled Preparing for the Future of Artificial Intelligence [9]. The report was a clear signal of the USA's interest in AI, and noted that unclassified research on AI was being managed through the Networking and Information Technology Research and Development program, with the support of several federal funding agencies. At the time of the report, federal investment in unclassified AI research was estimated to be $\$ 1.2$ billion (US) and it was recommended that future investment should focus on basic research and long-term, high-risk initiatives, as the private sector investment in R\&D would be limited. The 2016 National Artificial Intelligence Research and Development Strategic Plan [10] that accompanied the report set several objectives for federally funded AI research, such as ensuring effective human-AI collaboration, developing shared public datasets, and measuring and evaluating AI technologies through standards and benchmarks. That plan was updated in 2019 [11], evidence of the USA's continued interest in AI. In 2018, the White House hosted the "Artificial Intelligence for American Industry" [12] summit, which promoted a "free market approach to scientific discovery that harnesses the combined strengths of government, industry, and academia" and examined "new ways to form stronger public-private partnerships to accelerate AI R\&D". AI was included as a priority area in the FY19 budget, particularly funding for projects focused on transportation, healthcare, workforce training, and military applications.

Beyond the USA, there is appetite for AI in the rest of the region. For example, Canada became the first North American country to release a national AI strategy in 2017 and Mexico recently released AI research priorities and strategic outlines.

\subsection{Asia: AI in China develops quickly}

In July 2017, China issued its New-Generation Artificial Intelligence Development Plan [13]. This document set key targets for China to become a world leader in AI theory, technology, and application by 2030. China's first milestone is to reach globally-advanced AI levels by 2020. The three-year action 
plan specifically focuses on strengthening China's manufacturing capabilities and support systems and attracting and training a skilled AI workforce. China's ambition in AI is backed by government funding of more than $\$ 2$ billion (US) for major R\&D programs in 2018 [14] and a \$2.1 billion (US) investment into an AI technology park in Beijing. In addition to these investments, large datasets (consistent with the size of the Chinese population) and relatively relaxed data regulations have created an advantage for China. Chinese corporate giants such as Baidu, Alibaba, and Tencent are also focussed on AI research, alongside investment firms such as Sinovation Ventures [15], which established an AI Institute in 2016.

Beyond China, interest in $\mathrm{AI}$ in Asia is exemplified by various national-level document releases in recent years. In 2018, Think Tank NITI Aayog published a discussion paper setting the agenda for India's national AI strategy [16]. Japan unveiled its Artificial Intelligence Technology Strategy in March 2017 [17], while the South Korean government announced a five-year plan to invest in and strengthen AI research and development. AI plans and programs are in various stages of development in Malaysia, Singapore, and Taiwan.

\subsection{Europe: The European Commission seeks to unify AI frameworks across the region}

In April 2018, the European Commission (EC) outlined a three-pronged approach to AI:

- Increase public and private investment in AI.

- Prepare for socio-economic changes.

- Ensure an appropriate ethical and legal framework.

These strategies are supported by the EC's plans to increase AI research funding to $€ 1.5$ billion for the 2018-2020 period under the Horizon 2020 program. Per the commission, "this investment is expected to trigger an additional $€ 2.5$ billion of funding from existing public-private partnerships, for example, on big data and robotics [18]". The EC also advocated for a "European AI Alliance" to foster cooperation across member states. This collaborative spirit is also reflected in the Declaration of Cooperation on Artificial Intelligence [19] on issues such as research, socio-economic challenges, and legal and ethical frameworks signed by European Union (EU) members. The importance of AI to the EC is clearly articulated in the Joint Research Centre's 2018 AI report, which outlines a broad range of industrial, business, and research activities (including patenting, frontier research, venture capital, start-ups, and public-funded projects) [20]. The launch of an AI watch facility in December 2018, which functions to monitor and analyze AI developments, further exemplifies the importance of AI to the EC.

Several European member states have also published national AI strategies in recent years, resulting in a diversity of approaches in the region. France recently declared AI a national priority [21] and announced a strategic plan For a Meaningful Artificial Intelligence [22]. In March 2018, Italy released Artificial Intelligence at the Service of Citizens [23] and the German government released a national AI strategy in November 2018 [24]. The United Kingdom (UK) published its Industrial Strategy [25] in November 2017 and its Artificial Intelligence Sector Deal in April 2018 [26]. Other European countries that have recently released national strategies or reports on AI include Finland (Finland's Age of Artificial Intelligence [27]), Denmark (New Strategy to Make Denmark the New Digital Frontrunner [28]), and Sweden (National Approach for Artificial Intelligence [29]). Several Nordic and Baltic countries have also formed a regional collaboration in 2018 to develop AI capacity. 


\subsection{Interest in AI is a global phenomenon}

Strategic planning in AI is underway in several other countries [30]. For instance, the United Arab Emirates launched an AI strategy in 2017, the first country to do so in the Middle East. Russia has released research priorities and strategic outlines while Tunisia and Kenya have formed task forces to examine the development of AI in Africa. Together, these efforts underscore the growing recognition by individual countries and regions of the potential impact of AI on society and human life and the need to develop knowledge and expertise in this field.

\section{Conclusion}

Though AI's journey started over 60 years ago, the field has seen accelerated growth and technological advances in recent years that has increasing impact on various facets of our daily lives. Recent national and regional policies reflect the importance of competitiveness in the field with calls for large investments, as well as the need to address the tremendous change that AI will bring to human society and the ethical implications.

\section{About the Primary Author}

Dr. Bamini Jayabalasingham is a Senior Analytical Product Manager at Elsevier. Prior to joining Elsevier, she served as an AAAS Science and Technology Policy Fellow at the National Institutes of Health, where she supported implementation and evaluation efforts on policies for women's health research. She has provided advice on strategy development to support evidence-based policy and program formation for numerous public and private stakeholders including the Public Health Agency of Canada, the New York City Department of Public Health and Mental Hygiene, and Acidophil. She holds a BSc in Microbiology from the University of Toronto and a PhD in Molecular Microbiology and Immunology from Johns Hopkins University. Email: jayabalasingham@elsevier.com.

\section{References}

[1] Wikipedia. AI Winter. https://en.wikipedia.org/wiki/AI_winter, accessed September 8, 2019.

[2] Computer Vision. http://people.idsia.ch/ juergen/vision.html, accessed September 8, 2019.

- The NORB Object Recognition Benchmark. https://cs.nyu.edu/ ylclab/data/norb-v1.0/, accessed September 8, 2019.

- The CIFAR Image Classification Benchmark. http://www.cs.toronto.edu/ kriz/cifar.html, accessed September 8, 2019.

- The MNIST Handwritten Digits Benchmark. http://yann.lecun.com/exdb/mnist/, accessed September 8, 2019.

- The Weizmann \& KTH Human Action Recognition Benchmarks. http://www.nada.kth.se/cvap/actions/, accessed September 8, 2019.

- Fei Yin, Qiu-Feng Wang, Xu-Yao Zhang, and Cheng-Lin Liu, "Chinese characters from the ICDAR 2013 competition", 2013 12th International Conference on Document Analysis and Recognition, 2013. doi:10.1109/ICDAR.2013.218.

[3] J. Schmidhuber, Deep learning in neural networks: an overview, Neural Networks 61: (2015), 85-117 Historical overviews: https://doi.org/10.1016/j.neunet.2014.09.003; Review (survey) article: LeCun, Y., et al., Deep Learning, Nature, 2015; 521: 436-444. http://www.nature.com/articles/nature14539, accessed September 8, 2019.

[4] Eurasia Group, Sinovation Ventures. China embraces AI: a close look and a long view. December 2017. https://www. eurasiagroup.net/files/upload/China_Embraces_AI.pdf. 
[5] K.-F. Lee, AI Superpowers: China, Silicon Valley, and the New World Order. Houghton Mifflin Harcourt, New York, NY, 2018, https://aisuperpowers.com/about/about-the-book.

[6] Scopus is the world's largest source-neutral abstract and citation database curated by independent subject matter experts.

[7] Full methodology available on Elsevier's AI resource centre (https://www.elsevier.com/connect/resource-center/artificialintelligence). See in particular the methodology document (https://www.elsevier.com/?a=829143).

[8] T. Dutton, An Overview of National AI Strategies. Medium Politics + AI, 28 June 2018. https://medium.com/politics-ai/anoverview-of-national-ai-strategies-2a70ec6edfd, accessed September 8, 2019.

[9] Executive Office of the President National Science and Technology Council Committee on Technology. Preparing for the Future of Artificial Intelligence. October 2016. https://obamawhitehouse.archives.gov/sites/default/files/ whitehouse_files/microsites/ostp/NSTC/preparing_for_the_future_of_ai.pdf, accessed September 8, 2019.

[10] https://www.nitrd.gov/pubs/national_ai_rd_strategic_plan.pdf, accessed September 8, 2019.

[11] https://www.nitrd.gov/news/National-AI-RD-Strategy-2019.aspx, accessed September 8, 2019.

[12] The White House Office of Science and Technology Policy. Summary of the 2018 White House Summit on Artificial Intelligence for American Industry. 10 May 2018. https://www.whitehouse.gov/wp-content/uploads/2018/05/SummaryReport-of-White-House-AI-Summit.pdf, accessed September 8, 2019.

[13] The State Council. The People's Republic of China. China issues guidelines on artificial intelligence development. 20 July 2017. http://english.gov.cn/policies/latest_releases/2017/07/20/content_281475742458322.htm, accessed September $8,2019$.

[14] "China to spend over USD 2 billion in R\&D this year", The Economic Times, 7 January 2018. https://economictimes. indiatimes.com/news/international/business/china-to-spend-over-usd-2-billion-in-rd-thisyear/articleshow/62403032.cms, accessed September 8, 2019.

[15] Sinovation Ventures AI Engineering Institute. http://ai.chuangxin.com/, accessed September 8, 2019.

[16] NITI Ayog. Discussion Paper: National Strategy for Artificial Intelligence. June 2018. http://www.niti.gov.in/writereaddata/ files/document_publication/NationalStrategy-for-AI-Discussion-Paper.pdf, accessed September 8, 2019.

[17] Strategic Council for AI Technology, Artificial Intelligence Technology Strategy, March 2017, http://www.nedo.go.jp/ content/100865202.pdf, accessed September 8, 2019.

[18] European Commission. Artificial intelligence: Commission outlines a European approach to boost investment and set ethical guidelines, 25 April 2018. http://europa.eu/rapid/press-release_IP-18-3362_en.htm, accessed September 8, 2019.

[19] European Commission. EU Member States sign up to cooperate on artificial intelligence. 10 April 2018. https:// ec.europa.eu/digital-single-market/en/news/eu-member-states-sign-cooperate-artificial-intelligence, accessed September $8,2019$.

[20] A. Annoni, P. Benczur et al.Artificial Intelligence: A European Perspective, M. Craglia (ed.), Publications Office, Luxembourg, 2018.

[21] AI for Humanity. French strategy for artificial intelligence. https://www.aiforhumanity.fr/en/, accessed September 8, 2019.

[22] Artificial intelligence: Making France a leader. 30 March 2018. https://www.gouvernement.fr/en/artificial-intelligencemaking-france-a-leader, accessed September 8, 2019.

[23] The Agency for Digital Italy (AGID). Artificial Intelligence at the Service of Citizens. 2018. https://ia.italia.it/assets/ whitepaper.pdf, accessed September 8, 2019.

[24] AI Hub Europe. Exclusive: German AI-Strategy Paper in English. 26 July 2018. http://ai-europe.eu/exclusive-german-aistrategy-paper-in-english/, accessed September 8, 2019.

[25] HM Government. Industrial Strategy: Building a Britain Fit for the Future. 2017. https://assets.publishing.service.gov.uk/ government/uploads/system/uploads/attachment_data/file/664563/industrial-strategy-white-paper-web-ready-version.pdf, accessed September 28, 2019.

[26] Department for Business, Energy \& Industrial Strategy, Department for Digital, Culture Media \& Sport. Policy paper: AI Sector Deal. 26 April 2018. https://www.gov.uk/government/publications/artificial-intelligence-sector-deal/ai-sectordeal\# executive-summary, accessed September 28, 2019.

[27] Ministry of Economic Affairs and Employment. Finland's Age of Artificial Intelligence. 2017. http://julkaisut. valtioneuvosto.fi/bitstream/handle/10024/160391/TEMrap_47_2017_verkkojulkaisu.pdf, accessed September 8, 2019.

[28] Ministry of Industry, Business and Financial Affairs. New Strategy to Make Denmark the New Digital Frontrunner. 30 January 2018. https://eng.em.dk/news/2018/januar/new-strategy-to-make-denmark-the-new-digital-frontrunner/, accessed September 8, 2019.

[29] Government Offices of Sweden. National Approach for Artificial Intelligence. 2018. https://www.government.se/ information-material/2019/02/national-approach-to-artificial-intelligence/, accessed September 8, 2019.

[30] Dutton, T. An Overview of National AI Strategies. Medium Politics + AI. 28 June 2018. https://medium.com/politicsai/an-overview-of-national-ai-strategies-2a70ec6edfd, accessed September 8, 2019. 